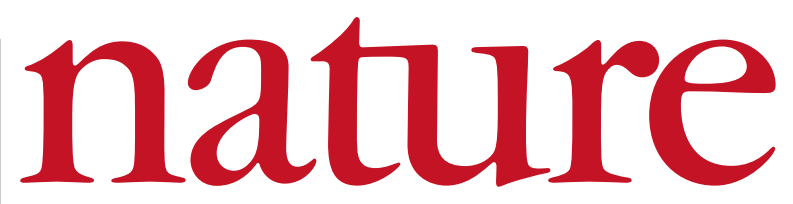

4 November 2004 Volume 432 Issue no 7013

\title{
Good eggs
}

There is no place for ageism in reproductive medicine.

E arlier this year, Harvard researchers turned dogma on its head by showing that, in mice, eggs are produced well into adult life (Nature 428, 145-150; 2004). This suggests that the menopause occurs not when eggs produced by the embryo eventually run out, as previously thought, but when cell death outpaces egg production in the adult ovary. If this is true for humans, the implications for treating infertility and health problems related to the menopause are profound. But it would be unfortunate if the suitability of older mothers also became a prominent part of discussions.

It is appropriate to scrutinize the societal impacts of any research, but a double standard may exist with regard to fertility. Men who father children into their golden years are more likely to receive back slaps than pressure to stop. In contrast, many fertility clinics deny services to women over 50, and even as young as 45 . The American Society for Reproductive Medicine's ethics committee discourages oocyte donation to postmenopausal women, citing a variety of concerns relating to the medical condition of mother and baby, as well as the psychological effect on the baby of having an older mother.

In truth, the likelihood that any individual will be a good parent depends on a variety of factors, only one of which is age. And while it is true that children of older parents have a higher risk of birth defects, this can be lessened by screening high-risk embryos for genetic defects before implantation. As for the medical risks, a 2002 study showed that pregnancy for women in their fifties from in vitro fertilization (IVF) is just as safe as for younger women who had IVF from donor eggs, and found no reason to exclude women over 50 from pregnancy on the basis of age alone (J.Am. Med. Assoc. 288, 2320-2323;2002).

Delaying the menopause is usually viewed in the context of fertility research, the subject of this week's Outlook (see page 37 ). Current research may help to prevent the debilitating conditions that arise when ovaries come to the end of their natural working lives. Even now, women spend nearly half their lives in menopause, an experience that can range from mild discomfort to debilitation. Osteoporosis is associated with loss of ovarian function, and there is evidence that cardiovascular disease, mood disorders, neurological problems and diminished sex drive are also connected. Genetic mouse models in which the menopause is delayed may eventually help disentangle which health risks are a direct consequence of menopause, and which are brought about by ageing (see Nature Genetics 21, 200-203; 1999).

Whether the menopause is seen as a medical condition requiring treatment or a phase of life to be tolerated, it is clear that, as human lifespan is extended, the ability to delay the menopause will be attractive to many women. Hormone-replacement therapy is no alternative to functioning ovaries, so it is to be hoped that a better understanding of ovarian regeneration will lead to improved treatments.

Research into women's reproductive health is important for its potential benefits to quality of life and overall health. It should not be sidetracked by detractors who make the patronizing and outdated argument that older women shouldn't have babies that they will be too old to care for.

\section{Bad funding}

The research of an environmental regulator is unlikely to win public trust if it relies on money from industrial lobby groups.

$\mathrm{T}$ he US Environmental Protection Agency (EPA) hasn't violated any laws by accepting US $\$ 2$ million from a chemical-industry lobby group to help it conduct toxicology research. But it is crossing a line that a regulator in the world's wealthiest nation should not have to.

As reported on page 6, environmental groups claim that accepting the funds represents a clear conflict of interest for the EPA, which is tasked with regulating companies that are members of the American Chemistry Council, the lobby group in question.

The study under scrutiny will examine the pathways by which young children are exposed to pesticides and household chemicals. The decision to accept the money is driven, EPA officials say, by a desire to conduct as comprehensive a study as possible, given constraints on the agency's research budget, which has remained essentially constant for almost a decade and is expected to dip slightly this year. The lobby group's cash will allow EPA scientists to analyse samples for the presence of potentially toxic compounds that they would otherwise have to leave out of the study. The agreement between the EPA and the chemistry council attaches no constraints, they add.

This is not a unique case: the EPA has struck agreements with dozens of companies to study a vast range of environmental problems over the past decade. But $\$ 2$ million is one of the largest sums ever accepted by the agency from an outside source and, in this case, the source has a direct interest in the outcome. Even if the wording of the agreement protects the research from any direct interference, it is hard for officials to argue that it doesn't give the appearance of compromising the agency's independence as a regulator.

Administrators at the agency may have little choice but to make deals with industry in order to move essential research forward, but there are ways to limit the appearance of impropriety. For example, industry could agree to give to a general research fund, rather than picking and choosing specific projects to support. Or it could partner universities or environmental groups on projects that all parties agree are important. Such moves would allay some of the concerns of environmentalists, and allow industry groups to support the science that they believe will ultimately lead to better regulation.

But above all, it is essential - not only for trust but also for adequate regulation - that the EPA's science budget be increased. The agency's mission requires it constantly to address questions of exposure and toxicity that are politically controversial, scientifically complex and poorly understood. It has been apparent for years that its research budget of about half-a-billion dollars a year is inadequate. Congress must give the cash-strapped agency the resources it needs to properly implement the plethora of mandates that it is meant to enforce. This idea is not so radical — it is supported by industry and environmental groups alike. 